\title{
Um Modelo Sensível ao Contexto para Avaliação da Saúde Mental por meio da Variabilidade da Frequência Cardíaca
}

\author{
Rodrigo Simon Bavaresco $^{1}$, Jorge Luis Victória Barbosa ${ }^{1}$ \\ ${ }^{1}$ Universidade do Vale do Rio dos Sinos (UNISINOS) \\ Av. Unisinos, 950 - Cristo Rei, São Leopoldo - RS, 93022-000 \\ rsbavarescolgmail.com, jbarbosa@unisinos.br
}

\begin{abstract}
The traditional method of mental health assessment, usually performed by a psychotherapist, shows relevant rates of inaccuracy. This work presents RevitalMe model, which analyzes the heart rate in order to contribute to the traditional method. The model provides daily information of the individual to psychotherapist, establishing a correlation between the mental health and the places frequented, through context-awareness. The evaluation of the model was performed with the implementation and use of a prototype applied to stress, which presents $\mathrm{F1-Score}$ of $88 \%$ in classification status of the individual between "stressed" and "not stressed". The perceived utility of the model is $83 \%$ according to 5 psychotherapists.
\end{abstract}

Resumo. O método tradicional de avaliação da saúde mental, realizado normalmente por um psicoterapeuta, mostra índices de imprecisão relevantes. Este trabalho apresenta o modelo RevitalMe, que analisa a frequência cardíaca a fim de contribuir ao método tradicional. O modelo proporciona ao psicoterapeuta informações do dia a dia do indivíduo, estabelecendo uma correlação entre a saúde mental e os lugares frequentados, por meio da sensibilidade ao contexto. A avaliação do modelo foi realizada com a implementação e uso de um protótipo aplicado ao estresse, que apresenta F1-Score de $88 \%$ na classificação do estado do indivíduo entre "estressado" e "não estressado". A utilidade percebida do modelo é de $83 \%$ de acordo com 5 psicoterapeutas.

\section{Introdução}

Segundo estimativas realizadas pelo World Health Organization [WHO 2017], mais de 300 milhões de pessoas no mundo sofrem com transtornos depressivos e 264 milhões sofrem com transtornos de ansiedade, o que representa respectivamente $4,4 \%$ e $3,6 \%$ da população mundial. Ambos podem ocorrer como resultado de não lidar adequadamente com um evento estressante na vida. Desta forma, o estresse mental também deve ser levado em consideração. Ele pode reduzir o desempenho diário do indivíduo, causar disfunções cognitivas, doenças cardiovasculares, depressão e conduzir à morte [WHO 2001].

Nas últimas décadas, novas informações dos campos da neurociência e da medicina têm avançado na compreensão do funcionamento mental. Elas indicam que transtornos mentais resultam da interação biológica (predisposição genética) com fatores psicológicos e sociais, e formam-se essencialmente no cérebro [WHO 2001]. No entanto, 
mesmo possuindo uma origem psicológica, transtornos mentais afetam diversos processos fisiológicos do corpo humano [Quintana et al. 2016].

A frequência cardíaca é um processo fisiológico que quando exposta a transtornos mentais sofre alteração. O Sistema Nervoso Autônomo (SNA) é um sistema que envolve-se na geração dessa excitação fisiológica [Rajendra Acharya et al. 2006]. O SNA é composto pelo Sistema Nervoso Simpático (SNS) e pelo Sistema Nervoso Parassimpático (SNP). No momento em que o SNA é desencadeado, o Sistema Nervoso Parassimpático (SNP) é suprimido e o Sistema Nervoso Simpático (SNS) torna-se ativo [Taelman et al. 2008]. Isso resulta na secreção dos hormônios epinefrina e norepinefrina no fluxo sanguíneo, que conduzem à mudanças na Frequência Cardíaca (FC) e na Variabilidade da Frequência Cardíaca (VFC).

Segundo Rajendra Acharya et al. (2006), conceitua-se a VFC como a variação ao longo do tempo entre batimentos cardíacos consecutivos. A VFC é normalmente obtida por meio da leitura dos sinais de um eletrocardiograma (ECG), com duração de minutos ou horas, e calculada a diferença entre duas ondas $\mathrm{R}$, denominando-se esta diferença de intervalos RR [Peltola 2012]. A fim de obter os índices da VFC, pode-se analisar instrumentos tais como o cardiofrequencímetro. Este instrumento encontra-se em dispositivos vestíveis - wearables - e apresenta resultado aceitável quando comparado ao ECG.

Para a avaliação de indivíduos que possam apresentar problemas com a saúde mental, o método tradicional realizado por um especialista, normalmente um psicoterapeuta, apresenta grandes índices de imprecisão [Webb and Parks 2016, Quintana et al. 2016, Choi et al. 2017]. Esta avaliação acontece por meio de uma análise cuidadosa de informações históricas do indivíduo e dos familiares, bem como entrevistas clínicas esquematizadas estruturalmente, onde o paciente informa seus sintomas e o nível do estado psicológico [WHO 2001]. De acordo com Webb e Parks (2016), este processo é subjetivo e pode ser afetado pelos próprios problemas de saúde mental do indivíduo, problemas de memória ou habilidades de articular o que está sendo vivenciado. Além disso, os pacientes podem relatar menores sintomas dependendo das consequências reais de um diagnóstico. Conforme Quintana, Alvares e Heathers (2016), podem existir dificuldades no diagnóstico preciso caso o entrevistador seja alguém inexperiente. Ademais, os diagnósticos obtidos através de questionários e auto-avaliações são os meios menos precisos para coletar informações, porque a maioria dos pacientes desconhece ou não consegue identificar corretamente o que é importante [Quintana et al. 2016, Choi et al. 2017].

Portanto, o modelo descrito neste artigo, denominado RevitalMe, apresenta como contribuições: (i) analisar a fisiologia como meio mais objetivo a fim de contribuir ao método tradicional; (ii) utilizar o potencial de dispositivos móveis para coletar dados do dia a dia com o uso da sensibilidade ao contexto e (iii) apresentar índices de saúde mental de um paciente para um psicoterapeuta, correlacionando-os com os lugares frequentados, para fornecer um conjunto de informações que auxiliem as decisões psicoterapêuticas. Salienta-se que estes objetivos vão ao encontro das colocações de Tal e Torous (2017). De acordo com os autores, o mundo encontra-se no início de uma era que deve prover novos conhecimentos e novas ferramentas para estimular o diagnóstico, tratamento, reabilitação e recuperação da saúde mental [Tal and Torous 2017].

Este artigo está organizado em seis seções. A seção 2 apresenta os trabalhos re- 
lacionados. A seção 3 descreve o modelo proposto, denominado RevitalMe. A seção 4 apresenta os aspectos da implementação do RevitalMe e na seção 5 descrevem-se os métodos de avaliação e os resultados. Por fim, na seção 6 apresentam-se as conclusões e os trabalhos futuros.

\section{Trabalhos Relacionados}

Esta seção apresenta uma revisão da literatura em busca dos trabalhos relacionados com o modelo proposto. Para esta revisão, considerou-se como principal referência um estudo sistemático previamente realizado pelo autor. Neste estudo, cujo objetivo foi mapear o estado da arte da computação ubíqua aplicada à fisiologia, enfatizando à saúde mental, buscaram-se artigos acadêmicos em 8 bases de dados na área da ciência da computação e da saúde. Desta forma, aplicaram-se critérios de inclusão e exclusão de artigos que, por fim, resultaram em 5 trabalhos. Estes trabalhos serão apresentados e comparados a seguir.

Tabela 1. Comparativo entre os trabalhos relacionados

\begin{tabular}{lcccc}
\hline & Ambiente & $\begin{array}{c}\text { Aspectos } \\
\text { Fisiológicos }\end{array}$ & Contexto & $\begin{array}{c}\text { Saúde } \\
\text { Mental }\end{array}$ \\
\hline [Mayya et al. 2015] & Laboratório & VFC & Não & Estresse \\
[Al Osman et al. 2014] & Real & VFC & Não & Estresse \\
[Hovsepian et al. 2015] & Real & VFC, Resp. & Não & Estresse \\
[Dobbins and Fairclough 2017] & Real & VFC & Apresenta & Ansiedade \\
[Gjoreski et al. 2016] & Real & FC, VFC, BVP, & Apresenta & Estresse \\
\hline
\end{tabular}

Mayya et al. (2015) apresentaram um trabalho para distinguir o estado das pessoas entre estressado e não estressado, ajudando o usuário a entender melhor os padrões de estresse ao longo do dia e a tomar as medidas apropriadas para gerenciar situações estressantes. No trabalho de Al Osman, Eid e El Saddik (2014) propôs-se um modelo de referência para monitoramento contínuo da fisiologia e transmissão de mensagens relacionadas com o estado mental do usuário. Os testes da aplicação foram realizados inicialmente em laboratório e posteriormente em um ambiente de trabalho.

Os autores Hovsepian et al. (2015) apresentaram um modelo para avaliação contínua do estresse. O modelo foi treinado utilizando dados coletados em laboratório e testado tanto em laboratório como durante atividades diárias. No trabalho realizado por Dobbins e Fairclough (2017), visou-se a criação de uma plataforma móvel para prover autoconhecimento aos usuários, monitorando ansiedade e raiva durante a atividade de dirigir veículos frente a situações reais. No trabalho de Gjoreski et al. (2016), apresentou-se um método para detecção contínua do estresse usando dados fornecidos por um dispositivo de pulso comercial. Além disso, os autores utilizaram ciência de contexto para compôr o método.

A fim de comparar os trabalhos relacionados, definiram-se 4 critérios como podese visualizar na Tabela 1. Portanto, os critérios de comparação são: (i) ambiente de aplicação, que sinaliza os trabalhos criados e testados ou diante de atividades reais do dia a dia ou em laboratórios; (ii) relação dos aspectos fisiológicos utilizados para computação, sendo obrigatório a presença da VFC; (iii) utilização de dados de contexto, considerado por Hovsepian et al. (2015) como relevante na tomada de decisão decorrente da fisiologia; (iv) saúde mental, evidenciando qual é a aplicação dos modelos propostos. A contribuição 
deste trabalho é composta pelos quatro critérios de comparação: analisa-se aspectos fisiológicos em ambientes reais utilizando sensibilidade ao contexto para inferir o estado psicológico de indivíduos, com o intuito de fornecer gráficos para psicoterapeutas.

\section{Modelo RevitalMe}

O RevitalMe propõe um modelo computacional com a finalidade de fornecer resultados do cotidiano de indivíduos para acompanhamento psicoterapêutico diante das terapias estabelecidas. Para alcançar tal finalidade, dois atores devem ser envolvidos na interação com o modelo: o psicoterapeuta, normalmente um psicólogo ou psiquiatra, e o paciente, um indivíduo adulto qualquer.

A primeira interação entre atores e modelo acontece em uma clínica, onde o modelo permite o vínculo de tratamento entre o paciente e o psicoterapeuta envolvido. $\mathrm{Na}$ clínica, o modelo RevitalMe permite que, a partir de um aplicativo para dispositivo móvel, denominado RevitalMe-Mobile, seja pareado um wearable com sensor de frequência cardíaca para obter dados fisiológicos tais como a FC e a VFC.

Em seguida, o paciente deverá selecionar localizações semânticas em conjunto com o profissional. A localização semântica refere-se à um lugar qualquer que faça parte do cotidiano do paciente, sendo nomeado por ele próprio. O profissional irá conduzí-lo a identificar lugares estratégicos que possam estar desencadeando problemas na sua saúde mental ou trazendo benefícios. Para tal atividade, o aplicativo RevitalMe-Mobile possui uma interface gráfica específica.

No decorrer da utilização do RevitalMe-Mobile, são formados históricos de contextos [Da Rosa et al. 2016], provenientes dos deslocamentos diários do paciente. Considerando-os como fonte de dados, o modelo é responsável por realizar processamento com objetivo de obter os índices de saúde mental, correlacionando-os com a localização semântica. Entende-se por índices de saúde mental o resultado psicofisiológico em valor numérico computado pelo modelo, proveniente da execução de um pipeline de aprendizado de máquina. Este resultado indica o percentual de tempo em que o paciente apresenta determinado estado psicológico, de acordo com a permanência na localização semântica.

A fim de apresentar os resultados ao psicoterapeuta, disponibiliza-se um website, denominado RevitalMe-Web, para realizar consultas no histórico de contextos. Assim, torna-se possível que o profissional acompanhe as atividades do paciente, realizando comparações diárias e entre localizações semânticas, dando importância aos índices de saúde mental computados, com o intuito de tomar decisões para as terapias estabelecidas.

\subsection{Arquitetura do Modelo}

Conforme pode-se visualizar na Figura 1, fazem parte da arquitetura três componentes: o aplicativo para dispositivo móvel denominado RevitalMe-Mobile, o website denominado RevitalMe-Web e o componente servidor, denominado RevitalMe-Server.

Cada componente possui responsabilidades distintas. O RevitalMe-Mobile coleta dados sensíveis ao contexto do paciente, provenientes de sensores do dispositivo móvel e do wearable, por meio de um agente de software. Pode-se citar como requisitos funcionais deste componente: cadastrar e autenticar usuário, cadastrar localização 
semântica, parear wearable, visualizar dados cardíacos e permitir vínculo terapêutico. $\mathrm{O}$ RevitalMe-Web mantém informações do psicoterapeuta e apresenta resultados computados pelo servidor para o profissional. Os requisitos funcionais são: cadastrar e autenticar profissional, solicitar vínculo ao paciente, consultar dados do paciente e controlar estado da leitura.

O RevitalMe-Server é responsável principalmente por receber dados do RevitalMe-Mobile, manter históricos de contextos, processar dados para o estado psicológico e fornecer consultas ao RevitalMe-Web. Na Figura 1, pode-se ver a interação entre os três componentes mencionados, bem como a definição das estruturas de software do RevitalMe-Server, subdivididos em camadas, módulos e agentes.

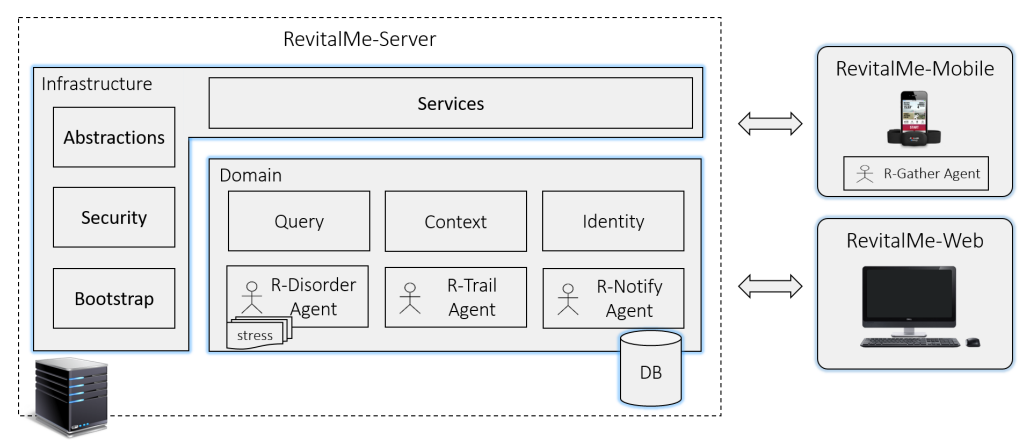

Figura 1. Arquitetura do modelo RevitalMe

As camadas Infrastructure e Domain provêm baixo acoplamento entre seus respectivos módulos. Os módulos de infraestrutura são quatro: Services, responsável por encontrar as Uniform Resource Identifiers (URI) dos módulos de domínio, expondo-as como serviços; Abstraction, modelado para que encapsule a complexidade de determinadas necessidades do servidor, abstraindo-as e reaproveitado código comum aos componentes; e Security, que fornece autenticação e autorização ao modelo. Por fim, o módulo Bootstrap é responsável por invocar métodos de inicialização de cada módulo de domínio bem como inicializar os agentes de software.

Os módulos de domínio são três: Identity, que visa manter as identidades de usuários e seus perfis, bem como os relacionamentos de vínculo terapêutico; e Context, que recebe em seus serviços os elementos de contexto referentes à entidade paciente, formando históricos de contextos. Desta forma, os elementos que compreendem o contexto são: a identidade do usuário, a localização, a data e a hora, a FC, a VFC, a atividade do usuário (parado, caminhando, correndo, de bicicleta e em veículo) e o estado da leitura. Além disso, este módulo apresenta serviços para manter localizações semânticas. Por fim, o módulo Query é responsável por receber as consultas que serão executadas no histórico de contextos e acionar o Agente R-Trail.

\subsubsection{Agentes do RevitalMe}

Nesta seção apresentam-se os agentes de software do RevitalMe. Os agentes foram modelados por meio da metodologia Prometheus, fazendo uso de um ambiente gráfico de 
modelagem denominado Prometheus Design Tool (PDT) ${ }^{1}$.

Escolheu-se utilizar agentes de software para modelar estes componentes pelas características que os mesmos apresentam, como a autonomia para execução de tarefas de acordo com as mudanças no ambiente a fim de cumprir determinados objetivos. Além disso, possuem características sociáveis, ou seja, para alcançar um objetivo em comum necessitam a interação com outros agentes, providas por meio de mensagens. Na Figura 2 pode-se visualizar a visão geral dos agentes do RevitalMe, onde cada agente é detalhado com suas ações, percepções e mensagens.

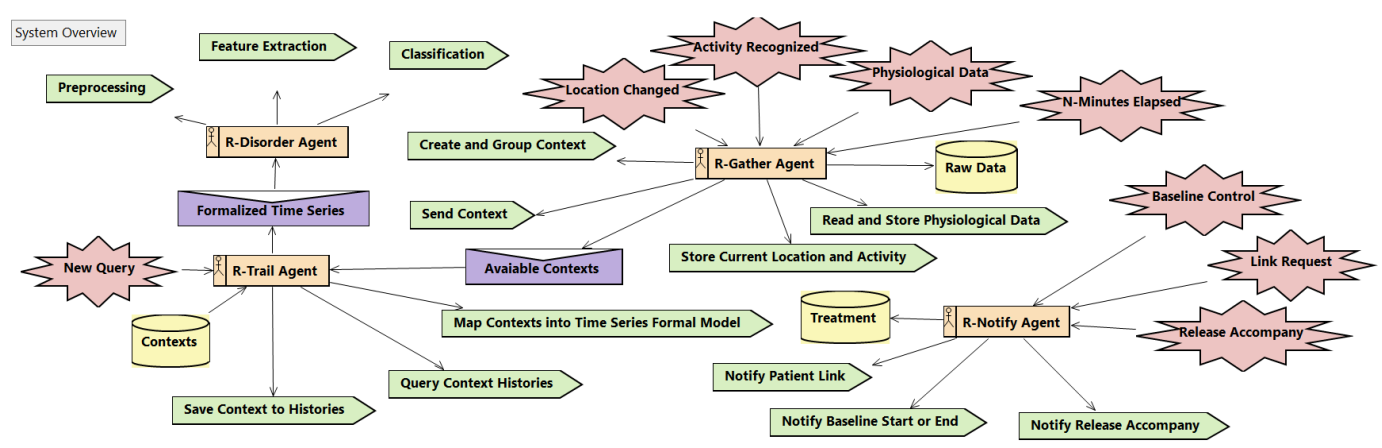

Figura 2. Diagrama da visão geral dos agentes do sistema

O Agente R-Gather é responsável por identificar mudanças no ambiente relacionadas com a atividade, localização, fisiologia e tempo, no decorrer da utilização do RevitalMe-Mobile por parte do paciente. O Agente R-Trail é responsável por identificar a chegada de novas mensagens ou a execução de uma nova consulta, com a finalidade de persistir registros de contextos ou recuperá-los, preparando-os para processamento dos índices da saúde mental. O Agente R-Disorder, por sua vez, deve realizar três etapas de processamento sob séries temporais extraídas do histórico de contextos, a fim de obter resultados numéricos que representem índices de saúde mental. As três etapas são: pré-processamento, extração de características e classificação. Elas caracterizam um pipeline de aprendizado de máquina. Este agente considera uma série temporal válida para processamento apenas se não possui atividade física, ou seja, apresentar a atividade "parado". Por fim, o Agente R-Notify deve identificar operações e eventos que aconteceram no modelo refente aos tratamentos entre o psicoterapeuta e o paciente, notificando-os.

\section{Aspectos da Implementação}

Com o intuito de avaliar o modelo RevitalMe, tornou-se necessário realizar a implementação de um protótipo. Portanto, codificaram-se os três componentes da arquitetura do modelo: RevitalMe-Mobile, RevitalMe-Web e o RevitalMe-Server.

Para o protótipo do RevitalMe-Mobile realizou-se a codificação de um aplicativo na plataforma Android compilado com a API mínima 19, que corresponde ao Android 4.4 (Kit Kat). No RevitalMe-Mobile, implementaram-se interfaces gráficas específicas para cada requisito funcional descrito anteriormente. Além disso, implementou-se o agente de software R-Gather. As percepções do agente foram codificadas como Android Services por possuírem três características: são operações de longa duração, monitoram elementos

\footnotetext{
${ }^{1}$ https://sites.google.com/site/rmitagents/software/prometheusPDT
} 
Tabela 2. Características no domínio de tempo e frequência

\begin{tabular}{|c|c|c|}
\hline Característica & Unidade & Fórmula \\
\hline \multicolumn{3}{|l|}{ Domínio de Tempo } \\
\hline Average of $R R$ interval & $m s$ & $\overline{R R}=\frac{1}{N} \sum_{j=1}^{N} R R_{j}$ \\
\hline Standard Deviation of RR intervals & $m s$ & $\sqrt{\frac{1}{N-1} \sum_{j=1}^{N}\left(R R_{j}-\overline{R R}\right)^{2}}$ \\
\hline Standard Deviation of the Average RR intervals & $m s$ & $\sqrt{\frac{1}{N-1} \sum_{j=1}^{N}\left(R R_{1_{j}}-\overline{R R_{1}}\right)^{2}}$ \\
\hline Root Mean Square of RR interval Diff. & $m s$ & $\sqrt{\frac{1}{N-1} \sum_{j=1}^{N}\left(R R_{j+1}-R R_{j}\right)^{2}}$ \\
\hline$\%$ of RR intervals that differ by more than $50 \mathrm{~ms}$ & $\%$ & $\frac{N N 50}{N-1} \times 100 \%$ \\
\hline \multicolumn{3}{|l|}{ Domínio de Frequência } \\
\hline Low Frequency $(\mathrm{LF})$ & $m s^{2}$ & Potência espectral entre 0,04 e $0,15 \mathrm{~Hz}$ \\
\hline High Frequency $(\mathrm{HF})$ & $m s^{2}$ & Potência espectral entre 0,15 e $0,4 \mathrm{~Hz}$ \\
\hline Ratio $L F / H F$ & $m s^{2}$ & Razão entre LF e HF \\
\hline
\end{tabular}

de hardware e realizam chamadas à APIs externas. Realizam-se todas estas operações em background, ou seja, sem a intervenção do paciente. Para verificar as mudanças de localização bem como o reconhecimento das atividades do usuário utilizou-se a API Google Location Services ${ }^{2}$. Para a leitura da frequência cardíaca e dos intervalos RR, codificou-se um Android Service para as conexões Bluetooth Low Energy do monitor cardíaco. Os intervalos RR foram lidos de forma contínua e com curta duração (shortterm) a cada 5 minutos [Quintana et al. 2016] e enviados ao RevitalMe-Server por meio de uma chamada à API RESTful, utilizando o protocolo HTTP.

Implementou-se o componente RevitalMe-Web como um website, fazendo uso do framework .Net Core na versão 2.0 com uso das linguagens C\# e Javascript, bem como HTML 5 e CSS 3 para construção das telas. Para o cadastro, a autenticação e a identificação do psicoterapeuta utilizou-se a API do Google Plus ${ }^{3}$.

Realizou-se a codificação do RevitalMe-Server com uso do framework .Net Core na versão 2.0 e na linguagem C\#. Os módulos de infraestrutura bem como os módulos de domínio foram codificados com a utilização de interfaces, classes abstratas e classes, de acordo com cada responsabilidade mencionada no modelo. No módulo Context, considerou-se um raio menor ou igual a 10 metros para identificar a relação de pertinência entre contextos e a localização semântica, com a utilização da fórmula de Haversine. Além disso, implementaram-se os agentes de software R-Trail e o R-Disorder, não sendo necessária a codificação do Agente R-Notify para validação do modelo, porque este apenas disponibiliza uma melhor usabilidade para os usuários. Ambos os agentes implementados foram codificados com o uso de classes, onde os percepts e mensagens dos agentes foram codificados como métodos das classes.

Implementou-se o Agente R-Disorder fazendo uso de classes na linguagem C\# que interoperam com scripts na linguagem R. Estes scripts foram codificados com uso da biblioteca $R H R V$. Para pré-processamento, codificou-se o agente para enviar uma série temporal de 5 minutos à um script na linguagem R. Este script computa a remoção dos artefatos fisiológicos ou técnicos dos intervalos [Peltola 2012]. Desta forma, codificou-se a remoção das batidas cardíacas que apresentam diferença no intervalo maior do que $10 \%$ da média dos últimos 50 intervalos. Além disso, considerou-se aceitável os valores da FC

\footnotetext{
${ }^{2}$ https://developer.android.com/training/location/

${ }^{3}$ https://developers.google.com/+/web/api/rest/
} 
com mínimo de 25 bpm e máximo de 180 bpm. Com o intuito de extrair características das séries temporais no domínio de tempo e no domínio da frequência, conforme a Tabela 2 , codificou-se outro script. As características no domínio de tempo foram obtidas por cálculos estatísticos, conforme apresentado. As características do domínio de frequência foram obtidas computando a Transformada Rápida de Fourier das séries de intervalos RR reamostrados de forma uniforme a $4 \mathrm{~Hz}$, utilizando interpolação linear.

Adicionalmente, para a etapa de classificação da saúde mental do indivíduo, implementou-se no Agente R-Disorder rotinas para classificar o nível de estresse. Desta forma, utilizou-se aprendizado de máquina para classificar entre o estado de "estressado" e o estado "não estressado", por meio de um modelo treinado com o algoritmo de classificação Support Vector Machine, o qual obteve o melhor desempenho dentre três classificadores, de acordo com testes estatísticos que foram realizados. Apresenta-se na próxima seção os testes estatísticos, os métodos de treinamento, validação e avaliação do modelo.

\section{Avaliação e Resultados}

A metodologia de avaliação e resultados está organizada em três perspectivas, conforme detalha-se a seguir.

\subsection{Computação dos Índices de Saúde Mental}

Nesta perspectiva busca-se avaliar o pipeline de aprendizado de máquina por meio do desempenho de classificação de três algoritmos para prever o estado psicológico como "estressado" ou "não estressado". Assim, esta avaliação nos permite descobrir o melhor dos três classificadores a serem usados na implementação do protótipo.

Os três classificadores utilizados na avaliação foram o Naive Bayes (NB), $K$ Nearest Neighbors (KNN) e Support Vector Machines (SVM). Eles foram escolhidos experimentalmente e treinados usando o mesmo pipeline de aprendizado de máquina apresentado anteriormente, isto é, pré-processamento e extração de características. A entrada de dados fisiológicos para treinamento e teste foi derivada do trabalho de [Healey and Picard 2005], presente na base de dados Physionet [Goldberger et al. 2000], onde foram mensurados fisiologicamente os níveis de estresse de 17 indivíduos em situação de condução de veículo.

Para cada indivíduo foram geradas séries temporais de 5 minutos com intervalos $\mathrm{RR}$, provenientes do ECG, rotulados de acordo com os resultados do trabalho de Healey and Picard (2005) (ground truth). Isto é, para a condição de repouso, neste trabalho, considerou-se como o estado "não estressado" e para as duas condições de condução (rodovia e cidade), considerou-se como o estado "estressado". Os dados totais foram divididos em dois segmentos, compreendendo $70 \%$ para o dataset de treino e $30 \%$ para dataset de teste. No experimento de treino foi realizado um grid search com validação cruzada ( $k$-fold cross validation) permitindo encontrar hiperparâmetros para cada classificador. No experimento de teste foi utilizado o modelo treinado com os hiperparâmetros e para avaliar a performance deste, a amostra desconhecida, ou seja, o dataset de teste, foi dado como entrada.

Com os resultados, calcularam-se métricas estatísticas para avaliar cada classificador: Sensitividade ou Recall, que se refere a identificar corretamente indivíduos sob es- 
tresse; Especificidade, que se refere a identificar corretamente os indivíduos que não estão sob estresse; Precisão, que é uma medida que nos diz qual a proporção de indivíduos que foram identificados como estressados e na verdade, estavam estressados e Acurácia, que é uma medida de previsões corretas de indivíduos estressados e não estressados. Por fim, também é apresentado o F1-Score, que é a média ponderada da Precisão e do Recall.

Tabela 3. Resultados estatísticos da avaliação dos três classificadores

\begin{tabular}{lrrrrr}
\hline Classificador & Sens. & Espec. & Prec. & Acc. & F1 \\
\hline Naive Bayes & 0.72 & 1.0 & 1.0 & 0.81 & 0.83 \\
K-Nearest Neighbors & 0.78 & 0.83 & 0.90 & 0.80 & 0.83 \\
Support Vector Machine & 0.88 & 0.75 & 0.88 & 0.84 & 0.88 \\
\hline
\end{tabular}

A Tabela 3 apresenta os resultados das métricas de cada classificador. Estes resultados mostram que, embora o SVM tenha a menor Especificidade, quando analisamos Acurácia, o SVM possui o maior valor. Além disso, procura-se um equilíbrio entre Recall e Precisão, então deve-se analisar o maior F1-Score. Nesse caso, o SVM também obteve o maior valor. Portanto, o SVM foi o melhor dos três classificadores nesta avaliação e foi usado no desenvolvimento do protótipo.

\subsection{Correlação dos Índices de Saúde Mental e Localização Semântica}

Para esta perspectiva foi realizado um teste de aceitação, denominado teste alfa, do RevitalMe. Desta forma, avaliou-se indiretamente os aspectos de modelagem e funcionalidades do sistema, por meio da utilização do protótipo durante 8 dias de um indivíduo.

Inicialmente, foi realizada a publicação do RevitalMe-Server no Azure, ambiente de Cloud Computing da Microsoft, e foi instalado o RevitalMe-Mobile no smartphone do indivíduo. O RevitalMe-Web foi publicado localmente e um vínculo terapêutico fictício foi realizado. Em seguida, o indivíduo cadastrou 5 localizações semânticas de três cidades distintas, pareou o wearable Polar $\mathrm{H7}$ e iniciou o monitoramento.

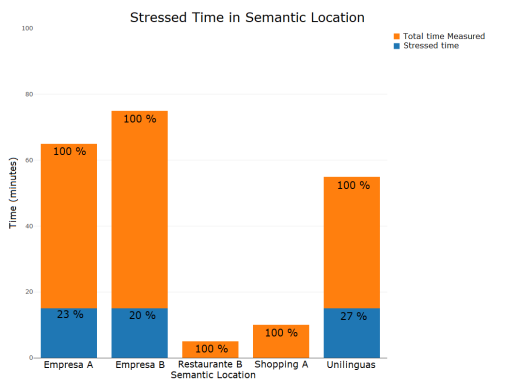

(a)

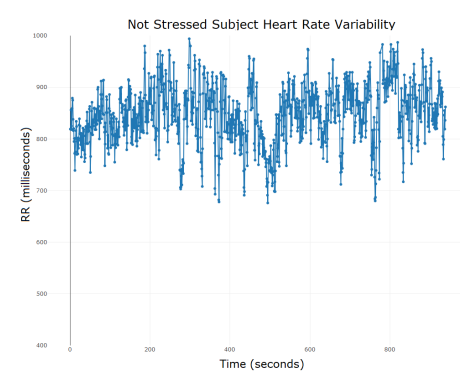

(b)

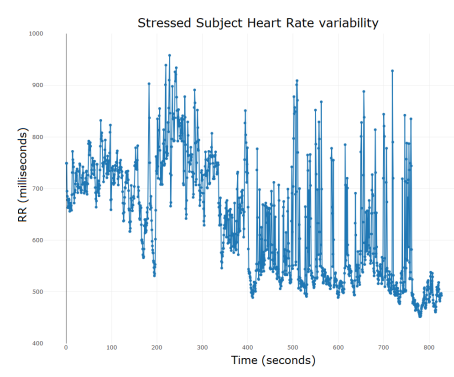

(c)

Figura 3. Gráficos provenientes da utilização do RevitalMe

Após a utilização, uma consulta da correlação dos índices de estresse e a localização semântica foi executada de forma online pelo RevitalMe-Web, e foi processada e gerada pelo RevitalMe-Server. Utilizou-se um filtro de data abrangendo um dos oito dias de uso do sistema, e a Figura 3a representa o resultado da consulta. Nela observa-se 
a correlação entre as 5 localizações semânticas frequentadas e os minutos de permanência nas mesmas, sendo evidenciado o percentual de tempo em que o indivíduo apresentou estresse. Além disso, a Figura $3 b$ ilustra um tacograma dos intervalos RR do indivíduo neste dia em horário que não apresentou estresse. Por outro lado, a Figura 3c ilustra um tacograma dos intervalos RR em horário que apresentou estresse. Ambos os tacogramas ilustram a real variação fisiológica coletada pelo protótipo em cada situação.

\subsection{Utilidade Percebida do RevitalMe}

Esta perspectiva foi criada para avaliar a contribuição que as ideias gerais do modelo podem fornecer para a tomada de decisão psicoterapêutica. Desta forma, para organizar esta perspectiva na busca por resultados baseou-se no Modelo de Aceitação de Tecnologia (TAM) [Marangunic and Granic 2015].

Por meio da aplicação de um questionário com 6 afirmações para 5 especialistas da área psicoterapêutica, buscou-se avaliar a utilidade percebida de acordo com o TAM, visto que os especialistas não utilizaram o sistema. Desta forma, foi disponibilizado uma descrição detalhada do modelo, englobando funcionalidades, objetivos e resultados direcionados totalmente para a área psicoterapêutica. As opções de respostas para as questões seguiram o padrão da escala Likert de cinco pontos, variando entre 1 (discordo totalmente) até 5 (concordo totalmente). As afirmações aplicadas por meio do questionário foram: (i) concordo que existe uma relação entre o estresse mental e os lugares que o indivíduo frequenta; (ii) a atividade de identificar lugares que o indivíduo frequenta induz a um diálogo sobre a rotina do mesmo, facilitando a compreensão sobre seus hábitos; (iii) os gráficos gerados poderão me ajudar no diagnóstico e no decorrer das terapias; (iv) ter conhecimento prévio às consultas sobre os níveis de estresse do indivíduo traria benefícios para minha tomada de decisão; (v) concordo que é possivel identificar uma melhora no quadro clínico do indivíduo por meio da comparação de dois gráficos gerados; (vi) mediante indicação, meus pacientes usariam o sistema. Ademais, uma pergunta com resposta discursiva foi aplicada aos especialistas: Quais os principais pontos positivos e negativos você pode observar no sistema?

Portanto, em relação a utilidade percebida, atingida por meio do agrupamento das respostas das 6 afirmações e apresentadas na Figura 4, é possível obter como resultado que $83 \%$ dos especialistas acreditam na utilidade do RevitalMe diante suas propostas, sendo de forma parcial ou total. Outros $10 \%$ dos especialistas discordam parcialmente e $7 \%$ acreditam ser indiferente.

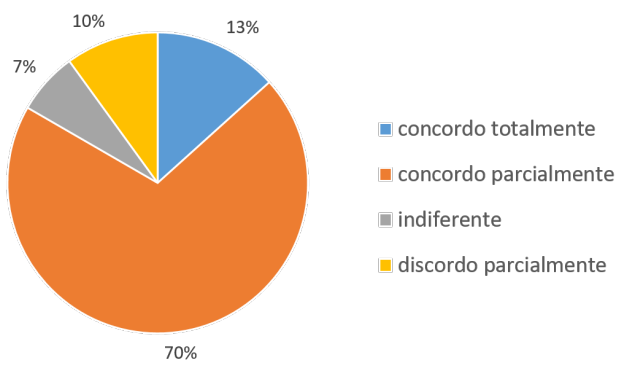

Figura 4. Utilidade Percebida do RevitalMe

No que diz respeito às respostas discursivas, pode-se elencar os pontos positivos descritos pelos especialistas, tais como: "aderência ao tratamento devido um re- 
curso interessante", "possibilidade de validar e reforçar os locais que provocam sensações agradáveis as pessoas", "facilidade na construção do diagnóstico e na avaliação da redução dos sintomas" e "objetividade na coleta de dados, uma vez que não dependese de aspectos do paciente que podem apresentar falhas, como a memória". Por outro lado, pode-se elencar os pontos negativos, tais como: "possível sensação de perseguição no paciente" e "possível 'boicote' de respostas pelo paciente". Salienta-se que o principal ponto negativo que foi relatado por um dos especialistas é: "monitorar o dia a dia do paciente não vai ao encontro com a técnica psicanalítica, que tem a associação livre como um dos pilares básicos". Por meio deste último relato, pode-se relacionar o resultado de $10 \%$ dos especialistas discordarem parcialmente e 7\% serem indiferente quanto as propostas do modelo, porque existem perfis de especialistas que apresentam atuações psicanalíticas.

\section{Conclusão}

Este trabalho apresentou o modelo RevitalMe, que auxilia a tomada de decisão dos psicoterapeutas com informações da saúde mental, provenientes do dia a dia dos pacientes. A performance do modelo foi medida pela acurácia de $84 \%$ e $\mathrm{Fl}$-Score de $88 \%$ para o melhor desempenho, utilizando o algoritmo SVM. Além disso, um teste de aceitação foi realizado no cotidiano de um indivíduo, contemplando 8 dias de uso. Este teste avaliou o potencial de correlacionar localizações semânticas com o estado psicológico do indivíduo.

Por fim, um questionário com 6 afirmações e 1 pergunta discursiva foi aplicado em 5 psicoterapeutas, para avaliar a proposta do RevitalMe em termos da sua utilidade. Por meio dos resultados, é possível concluir que as propostas do RevitalMe não se encaixam aos psicoterapeutas que seguem a linha teórica da psicanálise, visto que esta adota um método tradicional de associação livre. Por outro lado, as propostas são bem aceitas nos métodos da teoria cognitivo comportamental, porque podem utilizar o RevitalMe para estender ações e procurar resultados no dia a dia do paciente.

Para os trabalhos futuros, considera-se relevante melhorar o desempenho do algoritmo de classificação e aplicar testes em clínicas de psicoterapia com pacientes reais, visando obter dados que evidenciem a utilidade do RevitalMe na prática psicoterapêutica.

\section{Agradecimentos}

Os autores agradecem à FAPERGS, ao CNPq, à Coordenação de Aperfeiçoamento de Pessoal de Nível Superior (CAPES) - Código de Financiamento 001, e à Universidade do Vale do Rio dos Sinos (Unisinos) pelo apoio ao desenvolvimento desse trabalho.

\section{Referências}

Al Osman, H., Eid, M., and El Saddik, A. (2014). U-biofeedback: a multimedia-based reference model for ubiquitous biofeedback systems. Multimedia Tools and Applications, 72(3):3143-3168.

Choi, K.-H., Kim, J., Kwon, O. S., Kim, M. J., Ryu, Y. H., and Park, J.-E. (2017). Is heart rate variability (hrv) an adequate tool for evaluating human emotions? a focus on the use of the international affective picture system (iaps). Psychiatry Research, 251:192-196.

Da Rosa, J. H., Barbosa, J. L., and Ribeiro, G. D. (2016). Oracon: An adaptive model for context prediction. Expert Systems with Applications, 45:56-70. 
Dobbins, C. and Fairclough, S. (2017). A mobile lifelogging platform to measure anxiety and anger during real-life driving. In 2017 IEEE International Conference on Pervasive Computing and Communications Workshops (PerCom Workshops), pages 327-332.

Gjoreski, M., Gjoreski, H., Luštrek, M., and Gams, M. (2016). Continuous stress detection using a wrist device - in laboratory and real life. In Proceedings of the 2016 ACM International Joint Conference on Pervasive and Ubiquitous Computing Adjunct - UbiComp '16, pages 1185-1193. ACM Press.

Goldberger, A. L., Amaral, L. A. N., Glass, L., Hausdorff, J. M., Ivanov, P. C., Mark, R. G., Mietus, J. E., Moody, G. B., Peng, C.-K., and Stanley, H. E. (2000). Physiobank, physiotoolkit, and physionet : Components of a new research resource for complex physiologic signals. Circulation, 101(23):e215-e220.

Healey, J. and Picard, R. (2005). Detecting stress during real-world driving tasks using physiological sensors. IEEE Transactions on Intelligent Transportation Systems, 6(2):156-166.

Hovsepian, K., Al’Absi, M., Ertin, E., Kamarck, T., Nakajima, M., and Kumar, S. (2015). cstress: Towards a gold standard for continuous stress assessment in the mobile environment. In Proceedings of the 2015 ACM International Joint Conference on Pervasive and Ubiquitous Computing - UbiComp '15, volume 2015, pages 493-504. ACM Press.

Marangunic, N. and Granic, A. (2015). Technology acceptance model: a literature review from 1986 to 2013. Universal Access in the Information Society, 14(1):81-95.

Mayya, S., Jilla, V., Tiwari, V. N., Nayak, M. M., and Narayanan, R. (2015). Continuous monitoring of stress on smartphone using heart rate variability. In 2015 IEEE 15th International Conference on Bioinformatics and Bioengineering (BIBE), pages 1-5.

Peltola, M. (2012). Role of editing of r-r intervals in the analysis of heart rate variability. Frontiers in Physiology, 3:148.

Quintana, D. S., Alvares, G. A., and Heathers, J. A. J. (2016). Guidelines for reporting articles on psychiatry and heart rate variability (graph): recommendations to advance research communication. Translational Psychiatry, 6(5):e803-e803.

Rajendra Acharya, U., Paul Joseph, K., Kannathal, N., Lim, C. M., and Suri, J. S. (2006). Heart rate variability: a review. Medical \& Biological Engineering \& Computing, 44(12):1031-1051.

Taelman, J., Vandeput, S., Spaepen, A., and Van Huffel, S. (2008). Influence of mental stress on heart rate and heart rate variability. In IFMBE Proceedings, volume 22, pages 1366-1369.

Tal, A. and Torous, J. (2017). The digital mental health revolution: Opportunities and risks. Psychiatric Rehabilitation Journal, 40(3):263-265.

Webb, A. K. and Parks, P. D. (2016). Psychophysiological monitoring: An approach for the diagnosis and treatment of mental health disorders. IEEE Pulse, 7(1):31-34.

WHO (2001). The world health report 2001 - mental health: new understanding, new hope. Technical report.

WHO (2017). Depression and other common mental disorders: Global health estimates. Technical report. 